\title{
Spherical operator classification for coronary artery extraction
}

\author{
Chen Geng ${ }^{\mathrm{a}}$, Jian Yang ${ }^{\mathrm{b}}$, Yakang Dai ${ }^{\mathrm{a}}$, Zhaobang Liu ${ }^{\mathrm{a}}$ and Yuefang Dong ${ }^{\mathrm{a}}$ \\ ${ }^{a}$ Suzhou Institute of Biomedical Engineering and Technology, Chinese Academy of Sciences, Suzhou \\ 215163, China \\ ${ }^{b}$ Beijing Institute of Technology, Beijing 100086, China
}

\begin{abstract}
Computed tomography angiography (CTA) is a major noninvasive technology for imaging coronary artery disease, and effective and accurate vessel tracking method can help radiologists diagnose the disease more accurately. In this paper, a novel 3D vessel tracking method based on CTA data is presented. The method is comprised of preprocessing, a novel spherical operator, and hierarchical clustering, where the spherical operator consists of rays that are casted different directions in a spherical coordinate system. The vascular boundary is extracted by the spherical operator, and the tracking direction is also obtained by the hierarchical clustering. The method is evaluated with the Rotterdam Coronary Artery Algorithm Evaluation Framework. Results indicate that our method outperforms current state-of-the-art methods in terms of the overlap ratio on the vessel tracking of coronary arteries in CTA data.
\end{abstract}

Keywords: 3D vessel tracking, spherical operator, hierarchical clustering

\section{Introduction}

Computed tomography angiography, also called CTA, is a clinical examination method that combines the technology of conventional CT scan with that of traditional angiography. It is used as a noninvasive method for providing not only the anatomical form of the arteries but also the pathological information of arteries such as stenosis and occlusions. With the aid of robust vessel tracking algorithms, radiologists can evaluate the arteries more accurately [1]. However, fast and accurate extraction of coronary arteries is still a very challenging task. The most relevant vessel extraction techniques can be divided into two categories, i.e., automatic method and semi-automatic method. Automatic methods usually use the Hessian matrix and Frangi vessel filter [1,2]. The results have a high redundancy due to the complete automation. On the other hand, the results of semi-automatic method are more effective in clinic [1,2]. Many semi-automatic methods utilize a globoid operator to extract the vascular boundary, or use template matching as kernel function to segment the vessel areas. However, previously such methods have certain drawbacks in the continuity of tracking or clinical effectiveness [3-9].

\footnotetext{
${ }^{*}$ Corresponding author: Yakang Dai, Institute of Biomedical Engineering and Technology, Chinese Academy of Sciences, Suzhou 215163, China. Tel.: +86 0512 69588101; Fax: +86 0512 69588088; E-mail: daiyk@sibet.ac.cn.
}

0959-2989/14/\$27.50 @ 2014 - IOS Press and the authors. 


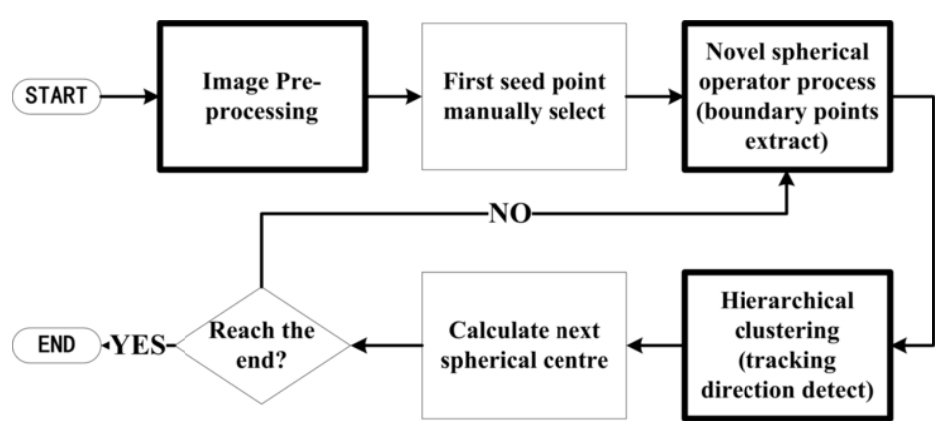

Fig. 1. Processing pipeline of the method.

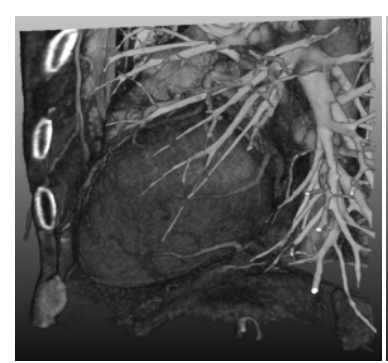

(a) Before preprocessing

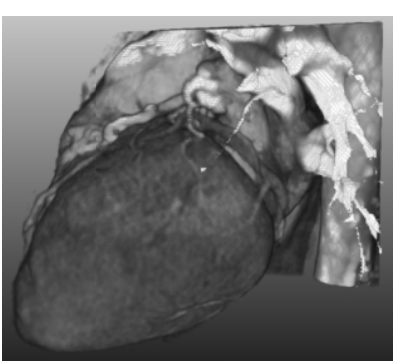

(b) After preprocessing

Fig. 2. Preprocessing result.

In this paper, a novel 3D vessel tracking method based on spherical operator and hierarchical clustering is presented. In Section 2, the processing pipeline and major steps of the method are described. Evaluation results of the proposed method are illustrated in Section 3. Conclusion is presented in Section 4.

\section{Approach}

Figure 1 shows the processing pipeline of our vessel tracking method. First, a multi-scale morphological closing operator is utilized to preprocess the CTA image to achieve better continuous vessel tracking. Then, with a seed point selected manually in the vessel, a novel spherical operator is used to detect the vessel boundary, and the proper direction of the blood vessel is obtained with hierarchical clustering. These steps will be described in the succeeding paragraphs.

\subsection{Pre-processing}

In this paper, a spherical dilation kernel whose radius is 3 voxels and a spherical erosion kernel with a 8 voxels radius are applied successively to the specified region with image intensities lower than a threshold. And a sigmoid filter with its function defined below is used to process the images, making a higher intensity contrast between coronary vessels and other tissues. 


$$
f(x)=(\operatorname{Max}-\operatorname{Min}) \cdot \frac{1}{\left(1+e^{-\frac{x-\beta}{\alpha}}\right)}+\operatorname{Min}
$$

In Eq. (1), the Max and Min stand for the maximum and minimum intensity of the CTA image, $\alpha$ and $\beta$ are two parameters of the sigmoid function, $x$ stands for the intensity of the voxels in the image. The results of pre-processing are shown in Figure 2. Obviously the coronary vascular tissue is enhanced in contrast with other tissues.

\subsection{Novel spherical operator}

We use a novel spherical operator to detect the vessel wall and meanwhile recognize the proper direction of the blood vessel. The novel spherical operator consists of rays casted from the centre to the surface, as presented in many other methods [7]. But the rays, as showed in Figure 3, have two opposite directions in couples along the diameters. And the diameters have a uniform angular difference of the polar angle and azimuthal angle within the spherical coordinate system.

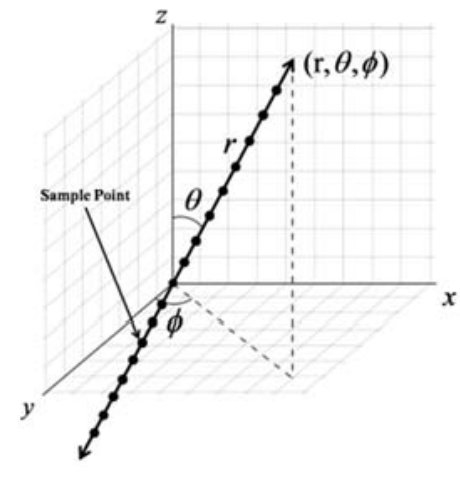

(a)

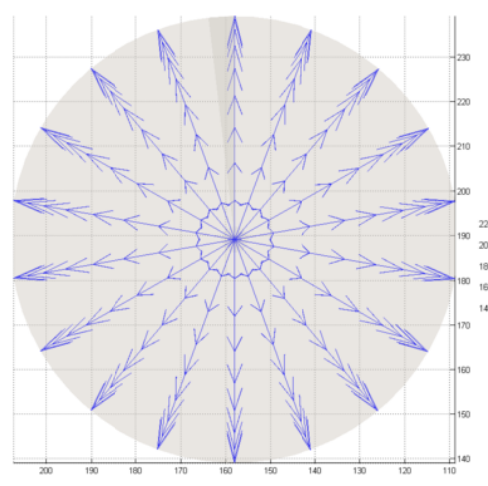

(b)

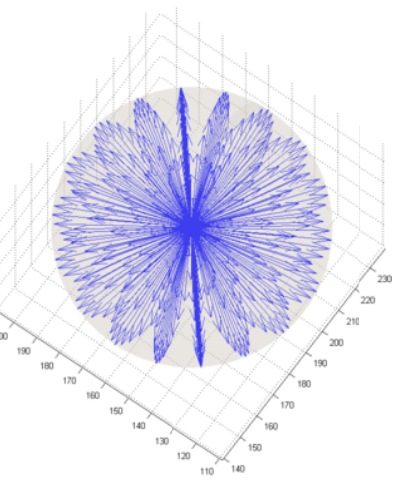

(c)

Fig. 3. The Novel Spherical Operator. (a) The diameter of the spherical operator with sample points. (b) The spherical operator in the top view. (c) The spherical operator in the $3 \mathrm{D}$ view.
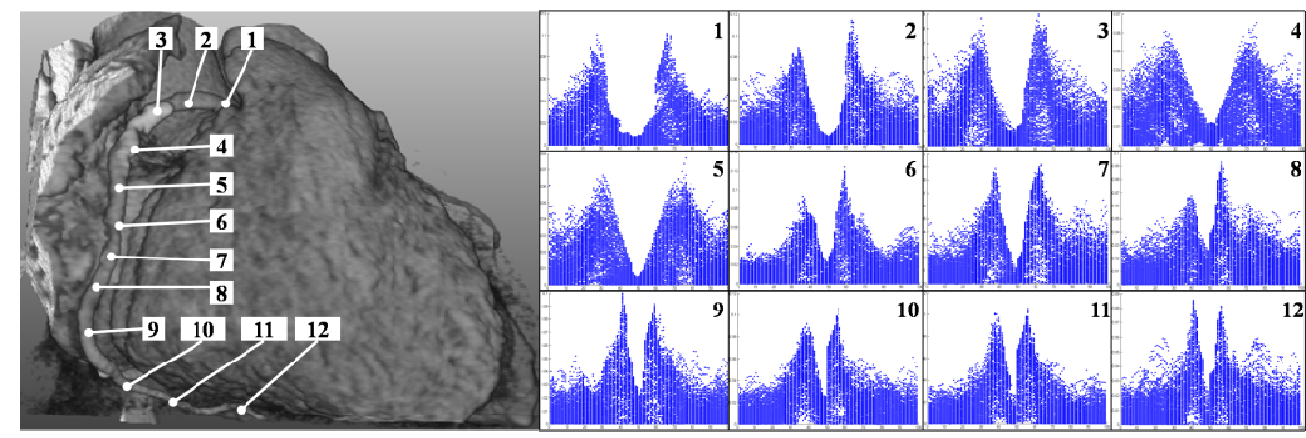

Fig. 4. Difference ratio scatter diagram along the coronary artery. 
The sample interval of the polar angle and azimuthal angle controls the number of rays, and in addition, affects the integrity of the extracted boundary. In this paper, the intervals are set to 10 degree and 20 degree respectively for the polar angle and azimuthal angle. Then the difference ratio based on image intensity is defined as Eq. (2), which indicates the intensity difference between the current position $\mathrm{P} 1$ and its neighboring position $\mathrm{P} 2$.

$$
\text { Ratio }=\left|\frac{I_{p 1}-I_{p 2}}{I_{p 1}}\right| \times 100 \%
$$

The ratio represents the difference between neighbors. In the spherical operator, there are many sample points along the rays. After casting rays in all diameters' two opposite directions, the difference ratio between two neighbors along all the pairs of rays is obtained. We perform the above processing along the coronary artery and make a diagram for every operator, in which the x-axis is the serial number of sample points along one pair of rays, and the $y$-axis is the difference ratio at the point, each point in the diagram represents one sample point on the diameter. Then we can obtain diagrams along the whole coronary artery (see Figure 4.).

As showed in Figure 4, there are always two peaks in the graph. In the experiments, it is proven that the sample points at peaks are the intersection points between rays and vascular wall. Taking one pair of rays, for example, it can be seen obviously from the difference ratio curve that whether the diameter intersects with the vascular wall (see Figure 5(a)). To get the peak points, 2D convex hull is constructed from the maximum points in the curve, and points at the top-left and top-right of the convex hull are the peak points. (see Figure 5(b)). All the points on the vascular wall could be obtained in this way.

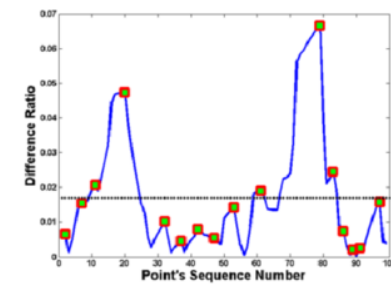

(a)

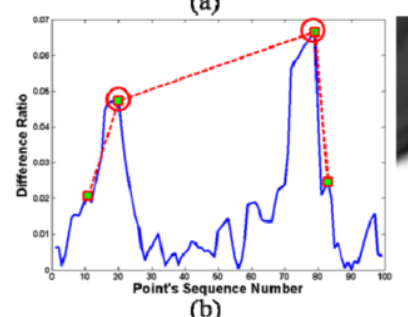

(b)

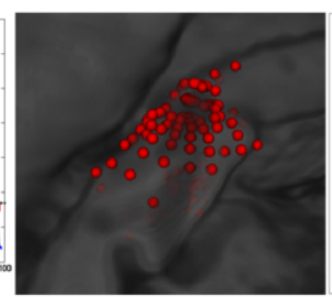

(c)

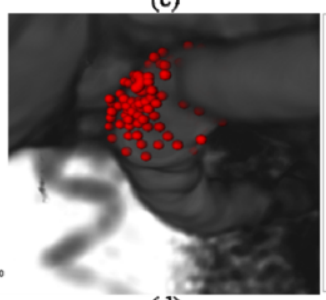

(d)

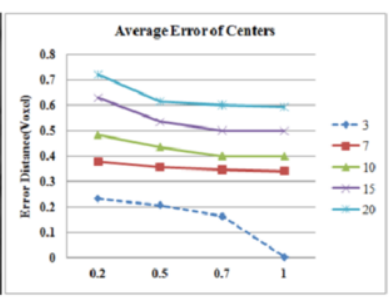

(c)

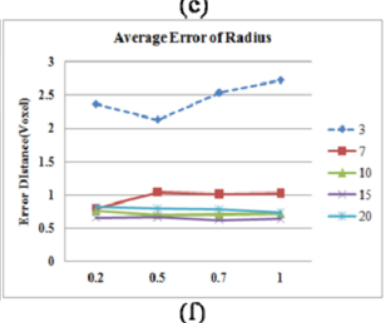

Fig. 5. Extract the boundary point using $2 d$ convex hull. (a) Get the maximum points along the difference ratio curve of a single diameter. (b) Construct a 2D convex hull with the maximum points above the average difference ratio. (c) and (d)The extracted boundary points in one spherical operator. (e) Average error of the extracted vessel centers. (f) Average error of the extracted vessel's radius. 
The sample distance and radius length have a significant impact on the error of extraction results. As showed in Figures 5(e) and 5(f), it's the average error of the spherical operator along the whole vessel. The $\mathrm{x}$-axis represents the sample distance in voxel, and y-axis represents the distance error. Different lines represents different radius. The average radius of the target vessel is 3.8 voxel, whose maximum is 7.5 voxel and minimum is 1.4 voxel. When the operator's radius is 3 voxel, more than half of the constructed spherical operators failed to recognize the vessel boundary, and the rest obtained a large error. In this paper, the radius is set to 15 voxel at the beginning of tracking, and the radius would adapt to the vessel automatically in the process of tracking.

\subsection{Hierarchical clustering}

In the previous step, the extraction result of the vascular boundary is obtained with the spherical operator. Since the spherical operator is constructed in the vessel, and its diameters are in uniform angular of the spherical coordinate system, when some of the rays intersect with the vessel wall, the other rays should point to the vessel direction. Classifying these ray direction vectors properly could contribute much in the orientation identification.

After excluding the noise diameters which intersects with the vascular wall but not be recognized, the residual diameters are pointing to the vessel direction. Due to the structure of vascular, if we take diameters' vectors as points with the same coordinate, then the points are in clumps. To classify these points, hierarchical clustering using the Euclidean distance is a suitable choice.

The single linkage law is well suited for classifying the features in clumps. Its recursion formula is defined as Eq. (3), which is the distance between class $i$ and class $j$.

$$
D_{i r}^{2}=D_{i p}^{2}-\frac{1}{2}\left|D_{i p}{ }^{2}-D_{i q}{ }^{2}\right|
$$

Then the classifying method is: using normalized direction vectors as samples, Euclidean distance between XYZ coordinate of vectors as the distance between samples, and hierarchical clustering as kernel algorithm. The general structure of the algorithm is:

initialization: classify samples $S_{i}(i \in[1, N])$ into Classes $C_{i}(i \in[1, N])$ with one sample per class

(1) take two random classes $C_{i}, C_{j},(i \in[1, N-1], j \in[i+1, N])$

(2) take random sample $S_{i p} \in C_{i}$ and random sample $S_{j p} \in C_{i}$

(3) calculate $D_{i j}=\min \left\{\right.$ Euclidean distance $\left.\left(S_{i p}, S_{j q}\right)\right\}$

(4) if $D_{i j}<$ Threshold

(5) $C_{j}$ falls into $C_{i}, C_{i}{ }^{\prime}=\left\{C_{i}, C_{j}\right\}$

(6) $C_{N}=\left\{C_{i}{ }^{1}, \ldots, C_{j-1}, C_{j+1}, \ldots, C_{N}\right\}$

(7) go to (1)

else

(8) terminate

For the coronary CTA images, there are always two or three classes left, which means a normal vessel or a bifurcation. After being processed by the algorithm, the vectors in Figure 6(b) are classified into two classes. By calculating the mean vector of each class, the result directions are obtained as 
showed in Figure 6(c). Furthermore, by calculating the angular difference between each direction and the previous direction, the proper direction for the next point can then be obtained easily. In addition, the next spherical centre is obtained according to Eq. (4). With a suitable step distance (generally smaller than half of the operator's radius), we can construct spherical operator repeatedly at the calculated centre, and finally extract the whole coronary vascular.

$$
\left(\mathrm{X}_{\text {next }}, \mathrm{Y}_{\text {next }}, \mathrm{Z}_{\text {next }}\right)=\left(\mathrm{X}_{\text {center }}+d \cdot \mathrm{V}_{x 1}, \mathrm{Y}_{\text {center }}+d \cdot V_{y 1}, \mathrm{Z}_{\text {center }}+d \cdot \mathrm{V}_{z 1}\right)
$$

\section{Results}

One of the extraction results of the CTA datasets is shown in Figure 7. The method presented in this paper is evaluated in the Rotterdam Coronary Artery Algorithm Evaluation Framework (RCAA) [1]. In the RCAA framework, extraction results are evaluated by three overlap values, which are overall overlap (OV), overlap until the first error (OF), and overlap with the clinically relevant part of the vessel (OT). The accuracy is measured by the mean distance to the standard centerlines. In addition, to make the extraction results comparable, the test dataset is chosen from the coronary CTA dataset supplied by the MICCAI conference [1].

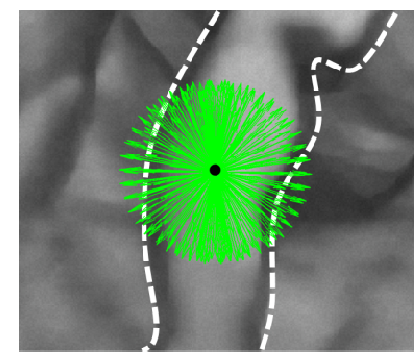

(a)

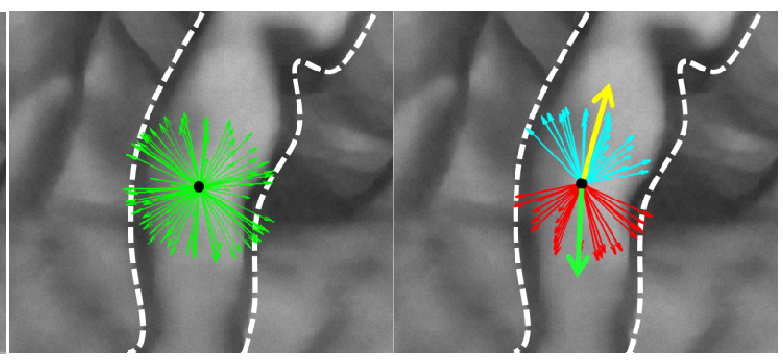

(b) (c)

Fig. 6. Classifying the vectors. (a) Construct a spherical operator. (b) Remove the diameters which intersect with the vascular boundary. (c) Refine the remaining diameters and classify them into two classes using hierarchical clustering.

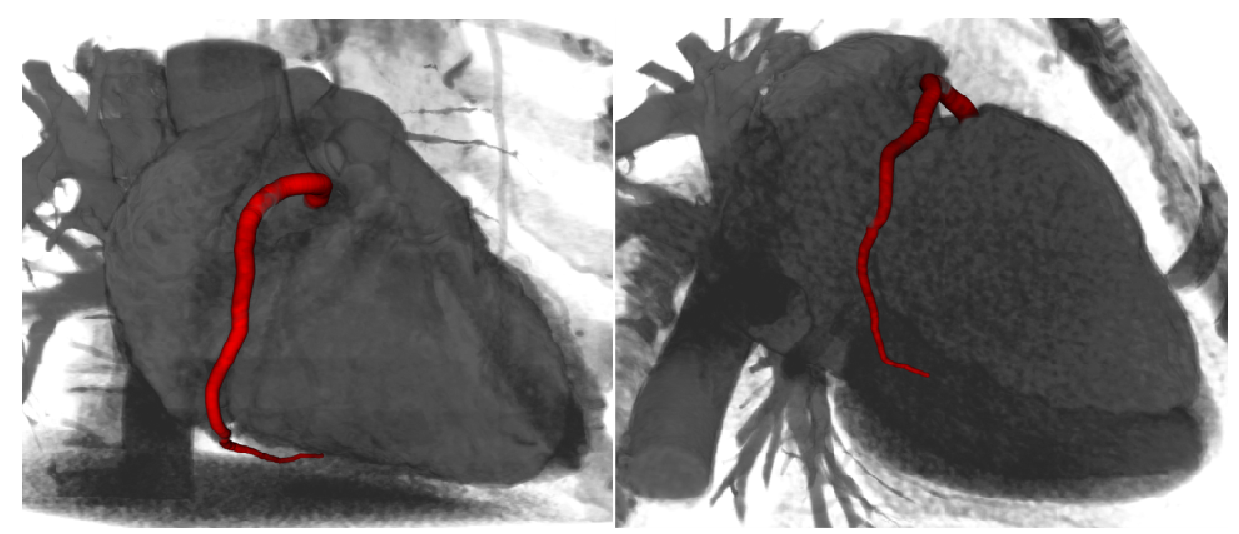

Fig. 7. Extraction results of the right coronary artery from different viewpoints. 
Table 1

Comparison results with other methods in the RCAA framework

\begin{tabular}{lllll}
\hline & OV $(\%)$ & OF $(\%)$ & OT $(\%)$ & Accuracy $(\mathrm{mm})$ \\
\hline Kitslaar & 78.8 & 64.4 & 81.2 & 0.29 \\
Wang & 75.6 & 56.1 & 78.7 & 0.39 \\
Zambal & 84.7 & 65.3 & 87.0 & 0.28 \\
Tek & 84.7 & 59.5 & 86.2 & 0.34 \\
Yang & 93.7 & 74.2 & 95.9 & 0.30 \\
This Paper & 86.9 & 73.7 & 98.3 & 0.39 \\
\hline
\end{tabular}

In this paper, the presented method is mainly compared with the methods proposed by Zambal [9], Wang [8], Kitslaar [4], Tek [5], and Yang [2]. After extracting the coronary arteries from 16 CTA datasets supplied by MICCAI conference, the experimental results are presented in Table 1. From the results, we can see that the method presented in this paper have the advantage in OV and OT. Our method's OV ranks 2nd with 86.9\%, and Yang's method ranks 1st in this category with $93.7 \%$. However, our method's OT ranks 1st with $98.3 \%$ among the six methods, $2.4 \%$ higher than Yang's method (ranks 2nd), whose OT is $95.9 \%$. In the OF and accuracy categories, our method also gets a good ranking, with the OF $73.7 \%$ and accuracy $0.39 \mathrm{~mm}$. Since the voxel size of the dataset is larger than the accuracy results of all the methods presented here, although the results of the methods differ, all of them achieve high accuracy.

\section{Conclusion}

In this paper, a novel method for tracking the coronary vascular tree from CTA image is presented. Our novel method consists of three main steps including image pre-processing, novel spherical operator for vessel boundary detection, and system clustering for direction judgment. The novel spherical operator consists of rays at different angles in the spherical coordinate system, and obtains the feature of vascular boundary with the difference ratio curve of voxel values between sample points on the rays. Quantitative evaluation was performed on the CTA dataset from MICCAI conference. The results of RCAA evaluation framework demonstrate good extraction ability and accuracy in the comparison with other methods. As the evaluation results show, advantage of our method is the overall overlap and overlap with the clinically relevant part of the vessel. The overlap until the first error indicates that our method has potential and we will continue improving. In conclusion, we present a novel vessel tracking method for the CTA image. Quantitative evaluation shows that our method can track the coronary vessel with high overlap and accuracy measurements. The novel vessel tracking method is potentially promising for both research and clinical use.

\section{Acknowledgement}

This work was supported in part by the Hundred Talents Program of CAS, NSFC grants (61301042, 61201117), and NSFJ grant (BK2012189). 


\section{References}

[1] J.M. Schaap, C.T. Metz, T. van Walsum, A.G. van der Giessen, A.C. Weustink, N.R. Mollet, C. Bauer, H. Bogunović, C. Castro and X. Deng, Standardized evaluation methodology and reference database for evaluating coronary artery centerline extraction algorithms, Medical Image Analysis 13 (2009), 701-714.

[2] G. Yang, P. Kitslaar, M. Frenay, A. Broersen, M.J. Boogers, J.J. Bax, J.H.C. Reiber and J. Dijkstra, Automatic centerline extraction of coronary arteries in coronary computed tomographic angiography, The International Journal of Cardiovascular Imaging (formerly Cardiac Imaging) 28 (2012), 921-933.

[3] J. Egger, T. Kapur, T. Dukatz, M. Kolodziej, D. Zukić, B. Freisleben and C. Nimsky, Square-cut: A segmentation algorithm on the basis of a rectangle shape, PloS One 7 (2012), e31064.

[4] P.H. Kitslaar, M. Frenay, E. Oost, J. Dijkstra, B. Stoel and J.H. Reiber, Connected component and morpholgy based extraction of arterial centerlines of the heart (CocomoBeach), The MIDAS Journal 9 (2008).

[5] H. Tek, M.A. Gulsun, S. Laguitton, L. Grady, D. Lesage and G. Funka-Lea, Automatic coronary tree modeling, The Insight Journal (2008)

[6] J.A. Tyrrell, E. di Tomaso, D. Fuja, R. Tong, K. Kozak, R.K. Jain and B. Roysam, Robust 3-D modeling of vasculature imagery using superellipsoids, IEEE Transactions on Medical Imaging 26 (2007), 223-237.

[7] B. Whited, J. Rossignac, G. Slabaugh, T. Fang and G. Unal, Pearling: Stroke segmentation with crusted pearl strings, Pattern Recognition and Image Analysis 19 (2009), 277-283.

[8] C. Wang and Ö. Smedby, Coronary artery segmentation and skeletonization based on competing fuzzy connectedness tree, in: Medical Image Computing and Computer-Assisted Intervention-MICCAI, Springer, New York, 2007, pp. $311-318$

[9] S. Zambal, J. Hladuvka, A. Kanitsar and K. Bühler, Shape and appearance models for automatic coronary artery tracking, The Insight Journal 4 (2008). 